\title{
Weighted One-norm Minimization with Inaccurate Support Estimates:Sharp Analysis via the Null-space Property
}

\author{
Mansour, H.; Saab, R.
}

TR2015-026 April 2015

\begin{abstract}
We study the problem of recovering sparse vectors given possibly erroneous support estimates. First, we provide necessary and sufficient conditions for weighted 11 minimization to successfully recovery all sparse signals whose support estimate is sufficiently accurate. We relate these conditions to the analogous ones for 11 minimization, showing that they are equivalent when the support estimate is $50 \%$ accurate but that the weighted 11 conditions are easier to satisfy when the support is more than $50 \%$ accurate. Second, to quantify this improvement, we provide bounds on the number of Gaussian measurements that ensure, with high probability, that weighted 11 minimization succeeds. The resulting number of measurements can be significantly less than what is needed to ensure recovery via 11 minimization. Finally, we illustrate our results via numerical experiments.
\end{abstract}

IEEE International Conference on Acoustics, Speech, and Signal Processing (ICASSP)

This work may not be copied or reproduced in whole or in part for any commercial purpose. Permission to copy in whole or in part without payment of fee is granted for nonprofit educational and research purposes provided that all such whole or partial copies include the following: a notice that such copying is by permission of Mitsubishi Electric Research Laboratories, Inc.; an acknowledgment of the authors and individual contributions to the work; and all applicable portions of the copyright notice. Copying, reproduction, or republishing for any other purpose shall require a license with payment of fee to Mitsubishi Electric Research Laboratories, Inc. All rights reserved. 



\section{WEIGHTED ONE-NORM MINIMIZATION WITH INACCURATE SUPPORT ESTIMATES: SHARP ANALYSIS VIA THE NULL-SPACE PROPERTY}

\author{
Hassan Mansour \\ Mitsubishi Electric Research Laboratories \\ Cambridge, MA 02139 \\ mansour@merl.com.
}

\begin{abstract}
We study the problem of recovering sparse vectors given possibly erroneous support estimates. First, we provide necessary and sufficient conditions for weighted $\ell_{1}$ minimization to successfully recovery all sparse signals whose support estimate is sufficiently accurate. We relate these conditions to the analogous ones for $\ell_{1}$ minimization, showing that they are equivalent when the support estimate is $50 \%$ accurate but that the weighted $\ell_{1}$ conditions are easier to satisfy when the support is more than $50 \%$ accurate. Second, to quantify this improvement, we provide bounds on the number of Gaussian measurements that ensure, with high probability, that weighted $\ell_{1}$ minimization succeeds. The resulting number of measurements can be significantly less than what is needed to ensure recovery via $\ell_{1}$ minimization. Finally, we illustrate our results via numerical experiments.
\end{abstract}

Index Terms - Compressed sensing, compressive sampling, sparse approximation, $\ell_{1}$ minimization, weighted $\ell_{1}$ minimization, null space property

\section{INTRODUCTION}

Consider an arbitrary $k$-sparse signal in $\mathbb{R}^{N}$ (i.e., a vector $x \in \mathbb{R}^{N}$ with at most $k$ non-zero entries) and consider its corresponding linear measurements $y \in \mathbb{R}^{m}$ with $m<N$, where

$$
y=A x .
$$

Compressed sensing $[1,2,3]$ deals with recovering $x$ from $y$, knowing $A$, using tractable algorithms. For example, in the compressed sensing context, the use of constrained $\ell_{1}$ norm minimization for the recovery of sparse signals is now standard, (see, e.g., $[1,2,3])$. One estimates $x$ via $x^{*}$, the minimizer of

$$
\min _{z}\|z\|_{1} \text { subject to } y=A z \text {. }
$$

This is primarily due to (2) lending itself to efficient convex optimization algorithms, and to the fact that when $A$ is chosen appropriately, all sufficiently sparse $x$ can be recovered
Rayan Saab

\author{
Department of Mathematics \\ The University of California, San Diego \\ La Jolla, CA 92093 \\ rsaab@ucsd.edu.
}

from $y$ by solving the $\ell_{1}$ minimization problem $[1,2,3]$. In particular, drawing the matrix at random from an appropriate distribution suffices, with high probability. For example, matrices whose entries are i.i.d. Gaussian random variables, work when ${ }^{1} m \gtrsim k \log (N / k)$.

One property of the measurement matrix $A$ that characterizes sparse recovery from compressive measurements is the null space property (NSP) (see, e.g., [4] ) defined below.

Definition 1. [4] A matrix $A \in \mathbb{R}^{m \times N}$ is said to have the null space property of order $k$ and constant $C$ if for any vector $h: A h=0$, and for every index set $T \subset\{1 \ldots N\}$ with $|T| \leq k$, we have

$$
\left\|h_{T}\right\|_{1} \leq C\left\|h_{T^{c}}\right\|_{1}
$$

In this case, we say that $A$ satisfies $\operatorname{NSP}(k, C)$.

A necessary and sufficient condition for the recovery of any $k$-sparse vector $x$ from $y$ using (2) is for the matrix $A$ to satisfy $\operatorname{NSP}(k, C)$ with $C<1$. Moreover, it can be shown (see, e.g., [5, Section 9.4]) using tools developed in $[6,7]$ and $[8]$ that Gaussian random matrices satisfy the null space property with probability greater than $1-\epsilon$ when $m>c k \ln e N / k$. Here, $c$ depends on $C$ and $\epsilon$, but the dependence is mild enough that $c \approx 8$ is a reasonable approximation when $N$ is large and $k / N$ is small. Such a bound is essentially sharp. If a matrix $A \in \mathbb{R}^{m \times N}$ guarantees recovery of all $k$-sparse vectors $x$ via $\ell_{1}$ minimization (2), then $m$ must exceed $c_{1} k \log \left(\frac{N}{c_{2} k}\right)$ for some appropriate constants $c_{1}$ and $c_{2}$ (see, e.g., [5, Theorem 10.11]).

Nevertheless, one may hope that in the presence of useful information (such as a relatively accurate estimate of the support of the sparse signal) that one may be able to significantly reduce the number of measurements needed to guarantee recovery. In fact, it is often the case in practice that signals exhibit structured support sets, or that an estimate of the support can be identified (for example audio signals are lowfrequency, the support of the transform domain coefficients of

\footnotetext{
${ }^{1}$ We write $x \gtrsim y$ when $x \geq C y$ for some constant independent of $x$ and $y$.
} 
videos does not change much from frame to frame). In this paper, we analyze a recovery method that incorporates support information by replacing (2) with weighted $\ell_{1}$ minimization. Given a support estimate $\widetilde{T} \subset\{1, \ldots, N\}$, we estimate $x$ via the minimizer of the optimization problem

$\min _{z} \sum_{i=1}^{N} w_{i}\left|z_{i}\right|$ s.t. $y=A z$, with $w_{i}=\left\{\begin{array}{cc}w \in[0,1], & i \in \widetilde{T} \\ 1, & i \in \widetilde{T}^{c}\end{array}\right.$

The idea behind (3) is to choose w : $=\left(w_{i}\right)_{i=1}^{N}$ such that the entries of $x$ that are "expected" to be large, i.e., those on the support estimate $\widetilde{T}$, are penalized less.

\subsection{Prior work}

Incorporating support estimates into algorithms for recovering signals from compressed sensing measurements has been studied in, e.g., [9, 10, 11, 12, 13, 14, 15, 16, 17, 18]. Vaswani and $\mathrm{Lu}[10,11,12]$ used a weighted $\ell_{1}$ minimization approach with zero weights on the known support. Their work derives sufficient recovery conditions that are weaker than the analogous $\ell_{1}$ minimization conditions of [2] when a large proportion of the support is known. Jacques [13] extended these results to deal with compressible signals and noisy measurements. Friedlander et al. [15] considered applying non-zero weights to the support estimate, further generalizing and refining the results of Vaswani and Lu. They provided tighter sufficient conditions for recovery, and their conditions depended on the accuracy and size of the support estimate. Subsequently, Mansour et al. [16] extended these results to the case when multiple support estimates with varying accuracies are available.

Using a different model for the support information, Khajehnejad et al. [14] derive sufficient recovery conditions for weighted $\ell_{1}$ minimization to recover sparse signals from compressed sensing measurements. In [14], one partitions $\{1, \ldots, N\}$ to two sets and the entries of $x$ supported on each set have a fixed probability of being non-zero, albeit the probabilities differ between the sets. Here the "support information" consists of knowing the partition and probabilities. Adopting the same model, Oymak et al. [17] derive bounds on the the minimum number of Gaussian measurements required for successful recovery when the optimal weights are chosen for each set. Their results are asymptotic in nature and pertain to the non-uniform model where one fixes a signal and draws the matrix at random. Here, every new instance of the problem requires a new draw of the random measurement matrix.

In this paper, we provide uniform recovery guarantees, i.e., the matrix is drawn once and successful recovery is guaranteed (with high probability) for all sparse signals when sufficiently accurate support information is available.

\subsection{Notation}

Throughout the paper, $\widetilde{T}$ is the support estimate used in (3). The size of $\widetilde{T}$ is $\rho k$ for some $\rho>0$ and the accuracy of $\widetilde{T}$ is $\alpha=\frac{|\widetilde{T} \cap T|}{|\widetilde{T}|}$. For an index set $S \subset\{1, \ldots, N\}$ we define

$$
\Gamma_{s}(S):=\left\{T \subset\{1, \ldots, N\}:\left|\left(S \cap T^{c}\right) \cup\left(S^{c} \cap T\right)\right| \leq s\right\} .
$$

We denote the restriction of the vector $x$ to the indices in $T \subset\{1, \ldots, N\}$ by $x_{T}$. Our results require that we introduce a weighted non-uniform null space property.

Definition 2. Consider the sets $T \subset\{1 \ldots N\}$ with $|T| \leq k$ and $\widetilde{T} \in \Gamma_{s}(T)$, and define $S=\left(\widetilde{T} \cap T^{c}\right) \cup\left(\widetilde{T}^{c} \cap T\right)$. A matrix $A \in \mathbb{R}^{m \times N}$ is said to have the weighted nonuniform null space property over the sets $T$ and $\widetilde{T}$ with constant $C$ if for any vector $h: A h=0$, we have

$$
\mathrm{w}\left\|h_{T}\right\|_{1}+(1-\mathrm{w})\left\|h_{S}\right\|_{1} \leq C\left\|h_{T^{c}}\right\|_{1},
$$

In this case, we say $A$ satisfies w-NSP $(T, \widetilde{T}, C)$.

We also require a uniform version of the above definition.

Definition 3. A matrix $A \in \mathbb{R}^{m \times N}$ is said to have the weighted null space property with parameters $k$ and $s$, and constant $C$ if for any vector $h: A h=0$, and for every index set $T \subset\{1 \ldots N\}$ with $|T| \leq k$ and $S \subset\{1 \ldots N\}$ with $|S| \leq s$, we have

$$
\mathrm{w}\left\|h_{T}\right\|_{1}+(1-\mathrm{w})\left\|h_{S}\right\|_{1} \leq C\left\|h_{T^{c}}\right\|_{1} .
$$

In this case, we say $A$ satisfies w-NSP $(k, s, C)$.

Thus, the standard null space property $\operatorname{NSP}(k, C)$ can be written as $1-\operatorname{NSP}(k, k, C)$. Moreover, we note that there should be no confusion between the notation used for the weighted non-uniform and uniform null space properties, as one pertains to subsets and the other to sizes of subsets.

\section{RECOVERY GUARANTEES VIA THE WEIGHTED NULL-SPACE PROPERTY}

Our first main result is Theorem $4 .^{2}$

Theorem 4. Given a matrix $A \in \mathbb{R}^{m \times N}$, every $k$-sparse vector $x \in \mathbb{R}^{N}$ is the unique solution to all optimization problems (3) with $\widetilde{T} \in \Gamma_{s}(\operatorname{supp}(x))$ if and only if $A$ satisfies $\mathrm{w}-$ $N S P(k, s, C)$ for some $C<1$.

In particular, the theorem states that if $A$ does not satisfy w-NSP $(k, s, C)$ for any $C<1$, then there exists a $k$-sparse vector $x$ supported on some set $T$ with $A x=y$ and a set

\footnotetext{
${ }^{2}$ Due to space limitations, we omit the proofs of our results. They can be found in a technical report available at http://www.math.ucsd.edu/ rsaab/publications.htm and will be included in a journal version of this manuscript.
} 
$\widetilde{T}$ with $\left|\left(\widetilde{T} \cap T^{c}\right) \cup\left(\widetilde{T}^{c} \cap T\right)\right| \leq s$ such that $x$ is not the unique minimizer of (3). On the other hand, if $A$ satisfies w-NSP $(k, s, C)$ for some $C<1$ then, every $k$-sparse vector $x$ is the unique minimizer of the optimization problem (3) provided $\widetilde{T}$ satisfies $\left|\left(\widetilde{T} \cap T^{c}\right) \cup\left(\widetilde{T}^{c} \cap T\right)\right| \leq s$. Thus recovery is guaranteed via (3) provided the support estimate is accurate enough.

The following proposition shows that weighted $\ell_{1}$ minimization can guarantee recovery even when $\ell_{1}$ minimization cannot recover all $k$-sparse signals.

Proposition 5. Let $A$ be an $m \times n$ matrix that satisfies $1-N S P\left(s, s, C_{s}\right)$ for some $C_{s}<1$ as well as $1-N S P(k-$ $\left.s, k-s, C_{k-s}\right)$ and $1-N S P\left(k, k, C_{k}\right)$ for some finite $C_{k-s}, C_{k}$. Then, A satisfies w-NSP $(k, s, C(\mathrm{w}))$, with

$$
C(\mathrm{w})=\frac{(1+\mathrm{w}) C_{s} C_{k-s}+C_{s}+\mathrm{w} C_{k-s}}{1-C_{s} C_{k-s}} .
$$

To see that Proposition 5 shows that weighted $\ell_{1}$ minimization can succeed where $\ell_{1}$ minimization fails, first note that if $C_{k}>1$ then there exists a $k$-sparse signal that cannot be recovered by $\ell_{1}$ minimization. Moreover, if one chooses $\mathrm{w} \leq \frac{1-2 C_{s} C_{k-s}-C_{s}}{C_{k-s}\left(C_{s}+1\right)}$ and if $C_{s}<\frac{1}{2 C_{k-s}+1}$ then $0 \leq C(\mathrm{w})<$ 1 , so Theorem 4 guarantees that $x$ is the minimizer of (3) whenever $\widetilde{T} \in \Gamma_{s}(T)$, where $T$ is the support of $x$. To further illustrate the relationship between $\ell_{1}$ minimization and its weighted counterpart, we present a corollary of Theorem 4 and Proposition 5.

Corollary 6. The weighted null space property w-NSP $\left(s, s, C_{s}\right)$ and the standard null space property 1-NSP $\left(s, s, C_{s}\right)$ are equivalent.

Corollary 6 implies that weighted $\ell_{1}$-minimization recovers all $s$-sparse signals $x$ from noise-free measurements $A x$ given a support estimate that is $50 \%$ accurate if and only if $\ell_{1}$ minimization recovers all $s$-sparse signals. To see this, note that w-NSP $\left(k, k, C_{k}\right), C_{k}<1$ guarantees via Theorem 4 that every $k$-sparse vector $x$ is the unique minimizer of (3) provided $\widetilde{T}$ satisfies $\left|\left(\widetilde{T} \cap T^{c}\right) \cup\left(\widetilde{T}^{c} \cap T\right)\right| \leq k$. Define $\rho:=\frac{|\widetilde{T}|}{k}$; since the accuracy of the support estimate, $\alpha:=\frac{|T \cap \widetilde{T}|}{|\widetilde{T}|} \geq$ $1 / 2$, then $\left|\left(T \cap \widetilde{T}^{c}\right) \cup\left(T^{c} \cap \widetilde{T}\right)\right|=(1+\rho-2 \alpha \rho) k \leq k$, so we are done.

\section{GAUSSIAN MATRICES}

Our second main result deals with matrices $A \in \mathbb{R}^{m \times N}$ whose entries are i.i.d. Gaussian random variables, which we refer to as Gaussian matrices. We establish a lower bound on $m$ above which these matrices satisfy the weighted null space property (with high probability), and hence, guarantee exact sparse recovery using weighted $\ell_{1}$ minimization. However, we begin with some simple observations to establish a rough lower bound on the number of measurements necessary for weighted $\ell_{1}$ minimization to guarantee exact recovery. Observe that $\mathrm{w}-\mathrm{NSP}(k, s, C)$ (with $k>s$ ) implies 1-NSP $(s, s, C)$, i.e., the standard null space property of size $s$ (this can be seen from the definitions of the properties). Consequently, w-NSP $(k, s, C)$ guarantees recovery of all $s$ sparse signals via $\ell_{1}$ minimization so it requires $m \geq c_{1} s \log \left(\frac{N}{c_{2} s}\right)$ (see, e.g., [5, Theorem 10.11]). This may lead us to hope that $m \approx s \log \frac{N}{s}$ suffices for recovery given an accurate support estimate because in weighted $\ell_{1}$ minimization $s$ plays the role of the size of the support estimate's error. However, even if one had a perfect support estimate, $k$ measurements would still be needed to directly measure the entries on the support. Combining these observations, we seek a bound on the number of measurements that scales (up to constants) like $k+s \log \frac{N}{s}$. We begin with a non-uniform result (Theorem 7) whose proof consists of a suitably modified version of the analogous proof for the standard null space property for Gaussian matrices [19], cf., [5], [8]. Again, the details are omitted for space considerations.

Theorem 7. Let $T$ and $\widetilde{T}$ be two subsets of $\{1, \ldots, N\}$ with $|T| \leq k$ and $\left|\left(T \cap \widetilde{T}^{c}\right) \cup\left(T^{c} \cap \widetilde{T}\right)\right| \leq s \leq k$ and let $A$ be a random matrix with independent standard Gaussian entries. Then A satisfies $\mathrm{w}-\operatorname{NSP}(T, \widetilde{T}, C)$ with probability exceeding $1-\epsilon$ provided

$$
\begin{aligned}
\frac{m}{\sqrt{m+1} \geq} & \sqrt{s+\alpha \rho k}+\left(\frac{1}{2 \pi e^{3}}\right)^{1 / 4} \sqrt{\frac{k}{\ln (e N / k)}} \\
& +C^{-1} \sqrt{2\left(\left(\mathrm{w}^{2}-2 \mathrm{w}(1-\alpha)\right) \rho k+s\right) \ln (e N / k)} \\
& +\sqrt{2 \ln \epsilon^{-1}}
\end{aligned}
$$

Importantly, the theorem suggests that given a particular support estimate accuracy $\alpha$ (which in turn governs the relationship between $s$ and $k$ ), one should optimize w to minimize the number of measurements in the theorem. Interestingly, the optimal choice satisfies $\mathrm{w}=1-\alpha$, which agrees with the intuition that a smaller weight should be applied when the confidence in the support estimate is high, and vice versa.

Moreover, one can use Theorem 7, combined with a counting argument and a union bound, to obtain a similar result which this time holds uniformly for all $k$-sparse signals supported on sets from $\Gamma_{s}(\widetilde{T})$.

Corollary 8. Let $\widetilde{T}$ be a subset of $\{1, \ldots, N\}$ and let $A$ be a random matrix with independent standard Gaussian entries. Then, with probability exceeding $1-\epsilon, A$ satisfies $\mathrm{w}-N S P(T, \widetilde{T}, C)$ for all sets $T \subset\{1, . ., N\}$ with $|T| \leq k \leq N / 2$ and $\left|\left(T \cap \widetilde{T}^{c}\right) \cup\left(T^{c} \cap \widetilde{T}\right)\right| \leq s \leq k$ provided

$$
\begin{aligned}
\frac{m}{\sqrt{m+1}} \geq & \left(1+\frac{1}{\left(2 \pi e^{3}\right)^{1 / 4} \sqrt{\ln (e N / k)}}\right) \sqrt{k+s} \\
& +C^{-1} \sqrt{2\left(\mathrm{w}^{2} k+s\right) \ln (e N / k)} \\
& +\sqrt{2 \ln \epsilon^{-1}+(s+1) \ln (e N / s)+k} .
\end{aligned}
$$


In the limiting case of large $m, N, k$ with small $k / N$ the condition (5) simplifies to

$$
\begin{aligned}
m \geq & \left(\sqrt{k+s}+C^{-1} \sqrt{2\left(\mathrm{w}^{2} k+s\right) \ln (e N / k)}\right. \\
& \left.+\sqrt{2 \ln \epsilon^{-1}+(s+1) \ln (e N / s)+k}\right)^{2},
\end{aligned}
$$

which reveals the benefit of using weighted $\ell_{1}$-minimization in reducing the number of measurements. In particular, taking $\mathrm{w}=0$ leads to the bound

$$
m \gtrsim k+\left(1+C^{-2}\right) s \ln (e N / s),
$$

which is essentially as good as one can hope for; one needs $k$ measurements to recover the non-zero entries even if the support was fully known, and about $s \ln (e N / s)$ measurements to recover the entries where the support estimate was erroneous. In particular, this bound can be significantly smaller than the analogous $m \gtrsim\left(1+C^{-1}\right)^{2} k \ln e N / k$ of standard $\ell_{1}$ minimization [5] when $s$ is significantly smaller than $k$, i.e., when the support estimate is very accurate.

\section{NUMERICAL EXPERIMENTS}

We present numerical simulations to illustrate our theoretical results. Throughout, we set $N=500$ and draw $m \times N$ matrices $A$ with i.i.d. standard Gaussian random entries, with $m$ ranging between 50 and 250 in increments of 25 . We generate $k$-sparse signals $x \in \mathbb{R}^{N}$, and vary $k$ between $\frac{m}{10}$ and $\frac{m}{2}$ in increments of $\frac{m}{20}$. The nonzero values in $x$ are drawn independently from a standard Gaussian distribution. We generate 50 instances of $A$ and $x$. For each instance, we compute the measurement vector $y=A x$ and compare the recovery performance of $\ell_{1}$ and weighted $\ell_{1}$ minimization. Specifically, support estimate sets $\widetilde{T}$ of size $k$ with accuracies $\alpha \in\{0.3,0.7\}$ are generated such that $\alpha k$ entries of $\widetilde{T}$ are chosen at random from the support of $x$. The remaining entries of $\widetilde{T}$ are chosen from outside the support of $x$. A weight $\mathrm{w}=1-\alpha$ is applied to the set $\widetilde{T}$ for weighted $\ell_{1}$ minimization.

We illustrate the performance of weighted $\ell_{1}$ minimization in Figs. 1 (a)-(b), corresponding to support estimate accuracies of $\alpha=0.3,0.7$. The solid red lines indicate the 0.85 empirical recovery rate thresholds for each of the weighted $\ell_{1}$ problems. Notice that the recovery thresholds of the weighted $\ell_{1}$ problems are shifted to the right compared to the standard $\ell_{1}$ recovery threshold (dashed red line) for values of $\alpha$ considered. Notice also that when $\alpha=0.7$, weighted $\ell_{1}$ minimization significantly outperforms standard $\ell_{1}$.

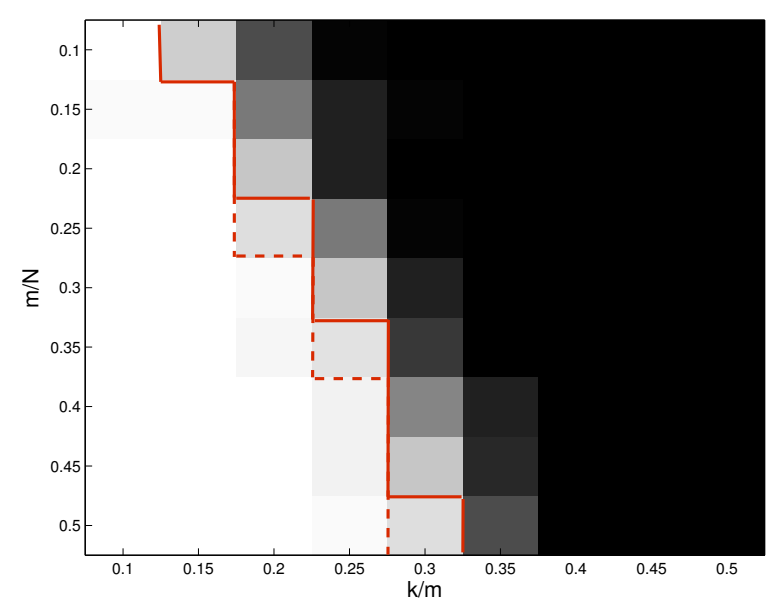

(a) $\alpha=0.3$

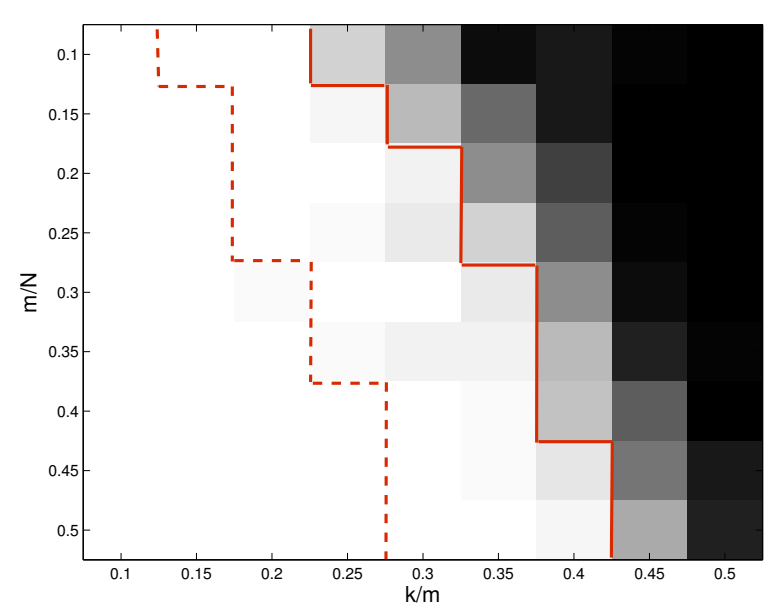

(b) $\alpha=0.7$

Fig. 1. Phase transition diagrams showing exact recovery rates using weighted $\ell_{1}$ minimization with weights applied to support estimate sets $\widetilde{T}$ with $\alpha=0.3,0.7$ and $\mathrm{w}=1-\alpha$. The dashed red line corresponds to the empirical 0.85 success rate for standard $\ell_{1}$ minimization. The solid red lines are the 0.85 rate thresholds for weighted $\ell_{1}$ minimization.

\section{REFERENCES}

[1] D. Donoho, "Compressed sensing.," IEEE Transactions on Information Theory, vol. 52, no. 4, pp. 1289-1306, 2006.

[2] E. J. Candès, J. Romberg, and T. Tao, "Stable signal recovery from incomplete and inaccurate measurements," Communications on Pure and Applied Mathematics, vol. 59, pp. 1207-1223, 2006.

[3] E. J. Candès, J. Romberg, and T. Tao, "Robust un- 
certainty principles: exact signal reconstruction from highly incomplete frequency information," IEEE Transactions on Information Theory, vol. 52, pp. 489-509, 2006.

[4] A. Cohen, W. Dahmen, and R. DeVore, "Compressed sensing and best k-term approximation," Journal of the American Mathematical Society, vol. 22, no. 1, pp. 211231, 2009.

[5] Simon Foucart and Holger Rauhut, A Mathematical Introduction to Compressive Sensing, Birkhauser Basel, 2013.

[6] Y. Gordon, “On milman's inequality and random subspaces which escape through a mesh in $\mathbb{R}^{n}$," in Geometric Aspects of Functional Analysis, Joram Lindenstrauss and VitaliD. Milman, Eds., vol. 1317 of Lecture Notes in Mathematics, pp. 84-106. Springer Berlin Heidelberg, 1988.

[7] M. Stojnic, "Various threshold for 11 optimization in compressed sensing," in available at arXiv:0907.3666, 2009.

[8] Venkat Chandrasekaran, Benjamin Recht, Pablo A. Parrilo, and Alan S. Willsky, "The convex geometry of linear inverse problems," Foundations of Computational Mathematics, vol. 12, no. 6, pp. 805-849, 2012.

[9] R. von Borries, C.J. Miosso, and C. Potes, "Compressed sensing using prior information," in 2nd IEEE International Workshop on Computational Advances in MultiSensor Adaptive Processing, CAMPSAP 2007., 12-14 2007, pp. $121-124$.

[10] Namrata Vaswani and Wei Lu, "Modified-CS: Modifying compressive sensing for problems with partially known support," IEEE Trans. on Signal Processing, vol. 58, no. 9, pp. 4595 - 4607, September 2010.

[11] N. Vaswani and Wei Lu, "Modified-CS: Modifying compressive sensing for problems with partially known support," in IEEE International Symposium on Information Theory, ISIT 2009, June 2009, pp. 488 - 492.

[12] Wei Lu and Namrata Vaswani, "Exact reconstruction conditions and error bounds for regularized modified basis pursuit," in Proc. of Asilomar Conf. on Signals, Systems and Computers, July 2010.

[13] Laurent Jacques, "A short note on compressed sensing with partially known signal support," Signal Processing, vol. 90, pp. 3308 - 3312, December 2010.

[14] M. Amin Khajehnejad, Weiyu Xu, A. Salman Avestimehr, and B. Hassibi, "Weighted 11 minimization for sparse recovery with prior information," in IEEE International Symposium on Information Theory, ISIT 2009, June 2009, pp. $483-487$.

[15] M.P. Friedlander, H. Mansour, R. Saab, and O. Yilmaz, "Recovering compressively sampled signals using partial support information," IEEE Transactions on Information Theory, vol. 58, no. 2, pp. 1122-1134, Feb 2012.

[16] H. Mansour and O. Yilmaz, "Weighted $\ell_{1}$ minimization with multiple weighting sets," in Proc. of the SPIE, Wavelets and Sparsity XIV, August 2011, p. 13 pages, (invited).

[17] S. Oymak, M.A Khajehnejad, and B. Hassibi, "Recovery threshold for optimal weight 11 minimization," in IEEE International Symposium on Information Theory Proceedings (ISIT), July 2012, pp. 2032-2036.

[18] Y. Wang and W. Yin, "Sparse signal reconstruction via iterative support detection," SIAM Journal on Imaging Sciences, pp. 462-491, 2010.

[19] M. Rudelson and R.Vershynin, "On sparse reconstruction from fourier and gaussian measurements," Communications on Pure and Applied Mathematics, to appear. Conference version in: CISS 2006 (40th Annual Conference on Information Sciences and Systems)., 2007. 